\title{
Paediatrics vascular injury management in resource limited setting: anaesthesia challenges
}

\author{
Muhammad Furqan Khan ${ }^{1}$, Syed Shabbir Ahmed ${ }^{1}$, and Khalid Samad ${ }^{1}$ \\ ${ }^{1}$ The Aga Khan University Hospital
}

November 6, 2021

\begin{abstract}
Vascular injuries with significant blood loss causing ischemia and revascularization for limb salvage are rarely done in developing countries. Hence, most hospitals and surgeons lack the experience to deal with vascular injuries. We report successful management of a 9 years child with left subclavian artery transection and massive blood transfusions.

Paediatrics vascular injury management in resource limited setting: anaesthesia challenges Authors:

1. Muhammad Furqan Khan: Resident, Aga Khan University Hospital, Anaesthesia Department. He was part of the team that managed the case and drafted the case report.

2. Syed Shabbir Ahmed: Senior Instructor, Aga Khan University Hospital, Anaesthesia Department. He was part of the team that managed the case and edited the case report.

3. Khalid Samad: Associate Professor, Aga Khan University Hospital, Anaesthesia Department. He was part of the team that managed the case and reviewed the case report.

Disclosure: There is no conflict of interest. The report is not funded by anyone and no funds have been acquired.Key Clinical Message:In emergency, waiting till optimization is not always possible. One needs to know the available resources and how to use them for the maximum advantage. Sometimes decisions have to be taken clinically even if they are not recommended.
\end{abstract}

\begin{abstract}
:
Vascular injuries with significant blood loss causing ischemia and revascularization for limb salvage are rarely done in developing countries. Hence, most hospitals and surgeons lack the experience to deal with vascular injuries. Here, we report successful management of a 9 years old child with left subclavian artery transection and massive blood transfusions.Key words: pediatric, vascular, anaesthesia, blood transfusion, revascularizationINTRODUCTION:Traumatic rupture of the subclavian artery is a rare lesion occurring in patients subjected to violent injury. Ischemia requiring surgical revascularization is also rare among pediatric population. ${ }^{1}$ Vascular injuries can result in significant blood loss, limb loss, serious life-long threatening disability, and even death. Injuries can result from a blunt or penetrating trauma, and these injuries can be isolated or in the setting of a complex multisystem trauma. ${ }^{2}$ Vascular trauma (i.e. arterial and venous) in the pediatric population is uncommon, as it occurs only in $0.6-1.4 \%$ of all pediatric injuries. ${ }^{3}$ Penetrating trauma is the most common vascular injuries affecting children. Arterial injury in children presents a challenge to the surgeon as well as to the anesthetist; pediatric patients are different from adults as they usually have atherosclerosis-free, spasm prone and smaller vessels, with small intravascular volume. ${ }^{4} \mathbf{C A S E}$ :A 9 years old male, weighing almost 20kg, presented in the Emergency room (ER) with stab wound in left supraclavicular region 12 hours before presentation. The patient had a deep stab wound accidentally at home. The patient started bleeding profusely from the wound and was rushed to the nearest hospital. Fluid resuscitation started with crystalloid, pressure applied on wound and the patient was immediately referred
\end{abstract}


to a tertiary care center from where he was further referred to paediatric tertiary care center. The patient presented there almost 4 hours after injury. On presentation his left upper limb was cold and there was no radial and brachial pulse palpable. There was a dull percussion note on left side and on auscultation no air entry. Chest X-ray was done on suspicion of hemothorax which showed left side white out lung field. He was maintaining airway, chest tube was inserted, which drained $1100 \mathrm{ml}$ of blood in 2 hours. The patient was further resuscitated with fluid and blood products (5 PCVs and 2 FFPs). Still, he developed hemorrhagic shock and collapsed for which he was intubated and ventilated but no CPR required. The patient was taken to Operating room (OR) and wound exploration done but unsuccessful and patient was referred to our hospital for vascular and cardiothoracic facilities.

On arrival (12 hours from injury), the patient was received intubated and on inotropic support (epinephrine at $0.08 \mathrm{mcg} / \mathrm{kg} / \mathrm{min}$ and dopamine at $10 \mathrm{mcg} / \mathrm{kg} / \mathrm{min}$ ) being given through peripheral IV line, laboratory tests were sent immediately, chest X-ray repeated and patient was rushed to OR with suspected left subclavian artery injury. The patient was put on anesthesia machine ventilator, maintaining hemodynamics with ionotropic support which was therefore continued same. Midazolam $2 \mathrm{mg}$, ketamine $40 \mathrm{mg}$ and cisatracurium $4 \mathrm{mg}$ given initially but no inhalational agent started as inotropes were being used. Right femoral A-line was inserted and sample for pre-operative ABGs drawn. CVP insertion was attempted in right femoral but was unsuccessful. Draping was completed and surgery started without undue delay of vascular access. After the start of surgery, ETT was changed under vision to $5.5 \mathrm{mmID}$ and fixed at $17 \mathrm{~cm}$. $18 \mathrm{G}$ cannula secured at right forearm. Pre-operative ABGs showed $\mathrm{pH} 7.06$, pCO2 56.30mmHg, pO2 157.30mmHg, HCO3 15.7mEq/L, $\mathrm{BE}-14.9 \mathrm{mEq} / \mathrm{L}$ and $\mathrm{SO} 297.50 \%$. Laboratory tests reported after start of surgery and showed $\mathrm{Hb} 11.3 \mathrm{~g} / \mathrm{dl}$, Hct $33.9 \%$, rest of the laboratory tests results were within normal limits except HCO3 13.9mmol/l, PT $>180$ s and INR $>18$. The patient continued to bleed which was further replaced with 3 PCVs, 6FFPs and 6 platelets. Cardiopulmonary bypass was arranged and kept on backup, to be used in case of need. Sternotomy was done immediately, injured site was identified and bleeding secured. Since sternotomy was done, arrangements were made to secure central access by surgeon directly if necessary. Till then, ionotropes were continued by peripheral access. As bleeding was immediately controlled, ionotropes started to get tapered and it was decided to establish central access at the end of surgery. Surgical findings revealed large hematoma in situ, at injured junction of left subclavian vein and internal jugular vein and transection of first part of subclavian artery distal to origin of vertebral artery. Almost 2 litres of blood and clots were evacuated from left thorax. Repair was done with PTFE interposition graft. The patient had almost 5 litres of blood intraoperatively. Intraoperative ABGs showed pH 7.29, pCO2 29.20mmHg, pO2 >480mmHg, HCO3 13.8mEq/L, BE -11.1mEq/L and SO2 100\%. There were multiple episodes of hemodynamic instability intraoperatively which were managed by adjusting infusion rates of epinephrine and dopamine and boluses of phenylephrine. The patient also received $600 \mathrm{mg}$ of calcium gluconate and $150 \mathrm{mEq}$ of NaHCO3. The surgery lasted for 5.5 hours. In summary, the patient received 5 PCVs, 2 FFPs, 1 litre colloid, 1 litre crystalloid preoperatively, while 3 PCVs , 6 FFPs, 6 Platelets, 1 litre crystalloid intraoperatively. Urine output was almost $50 \mathrm{ml} / \mathrm{hr}$ (total $300 \mathrm{ml}$ ) and he had a blood loss of almost 5 litres. Postoperative ABGs showed $\mathrm{pH} 7.38$, pCO2 37.50mmHg, pO2 121.20mmHg, $\mathrm{HCO} 321.50 \mathrm{mEq} / \mathrm{L}, \mathrm{BE}-3.5 \mathrm{mEq} / \mathrm{L}$ and $\mathrm{SO} 298.7 \%$. Left femoral CVP was inserted postoperatively and the patient was shifted to PICU for further management. His CBC sent from PICU after initial management showed $\mathrm{Hb} 8.1 \mathrm{~g} / \mathrm{dL}$, Hct $23.3 \%$ and platelets $116,000 \mathrm{X} 10^{6} / \mathrm{L}$. The patient was extubated after 30 hours, and discharged from hospital after 72 hours of surgery in stable condition.

DISCUSSION: Paediatric thoracic trauma itself is a unique challenge owing to the peculiar characteristics of anatomy, physiology, metabolism and therapeutic options in children. ${ }^{5}$ Penetrating trauma occurs nearly 6 -fold less than blunt trauma in children, while penetrating thoracic wounds even less. $35 \%$ of such patients require surgical intervention. ${ }^{5}$ In our patient the injury resulted in hemorrhagic shock. Also children develop hypoxemia more rapidly due to diminished FRC which gets deteriorated in case of chest trauma. Therefore, targets of anesthetic management were to prevent any physiological injury due to shock, minimizing hypoxia and hypoxemia and maintaining perfusion. ${ }^{5}$ Haemothorax due to thoracic vascular trauma leads to significant blood loss, hypovolemic shock, lung volume loss, empyema and possible lung entrapment. Chest tube 
placement for haemothorax may lead to further blood loss due to loss of tamponade effect on the injured vessel. ${ }^{6}$ This leads to severe haemodynamic alterations and warrants emergent surgical exploration of the wound. In addition to this, arterial trauma in children, also remains a significant challenge. When this occurs within thoracic cavity, it further aggravates the situation. Such injury usually results in massive blood loss leading to massive transfusion. ${ }^{6}$ In our case, massive transfusion had already been done before arrival and had lead to known complications which were coagulopathy, hypothermia and citrate toxicity causing hypocalcemia. Still, during surgery, further transfusion was necessary for maintaining hemodynamic stability and end organ perfusion along with managing the complications of massive transfusion. Another important objective was prevention of lethal triad of coagulopathy, acidosis and hypothermia. ${ }^{6}$ Since CVP could not be secured therefore permissive hypotension was tolerated. A balanced blood transfusion strategy of delivering PCV, FFPs and platelets was employed. Hypothermia and hypocalcemia were also managed by active warming, blood warmer and giving calcium. With the collaboration of anesthetic and surgical team, such a difficult case was managed quite successfully and the patient's life and arm were saved. Author: Muhammad Furqan Khan Resident, Aga Khan University Hospital furqandrmfk@gmail.com Co-author: Syed Shabbir Ahmed Senior Instructor, Aga Khan University Hospital shabbir.ahmed@aku.edu

\section{References:}

1. Harris LM, Hordines J. Major vascular injuries in the pediatric population. Ann Vasc Surg. 2003;17(3):266-269.

2. Grigorian A, Wilson SE, de Virgilio C, et al. Humerus fracture and combined venous injury increases limb loss in axillary or subclavian artery injury. Vascular. 2019;27(3):252-259.

3. Wahlgren CM, Kragsterman B. Management and outcome of pediatric vascular injuries. J Trauma Acute Care Surg. 2015;79(4):563-567.

4. Nystrup KB, Stensballe J, Bøttger M, et al. Transfusion therapy in paediatric trauma patients: a review of the literature. Scand J Trauma Resusc Emerg Med. 2015 Feb 15; 23: 21.

5. Minervini, F., Scarci, M., Kocher, G.J., Kestenholz, P.B. and Bertoglio, P., 2019. Pediatric chest trauma: a unique challenge.

6. St Peter SD, Ostlie DJ. A review of vascular surgery in the pediatric population. Pediatr Surg Int. 2007;23(1):1-10. 